\title{
Uso de esteroides anabolizantes por frequentadores de academias: Motivos e perspectivas
}

\author{
Use of anabolic steroids by gymgoers: Reasons and perspectives \\ Uso de esteroides anabólicos por parte de los aficionados al gimnasio: Razones y perspectivas
}

Recebido: 04/05/2021 | Revisado: 11/05/2021 | Aceito: 12/05/2021 | Publicado: 28/05/2021

Claudiana Marcela Siste Charal

ORCID: https://orcid.org/ 0000-0001-9977-2649

Universidade Estadual de Maringá, Brasil

E-mail: claudiana_siste@ hotmail.com

Fernando Carlos Messias Freire

ORCID: https://orcid.org/0000-0002-7812-9707

Universidade Estadual de Maringá, Brasil

E-mail: fcmfreire@uem.br

Eduardo Gauze Alexandrino

ORCID: https://orcid.org/0000-0002-4042-4954

Unicesumar, Brasil

E-mail: eduardogauze@ hotmail.com

Danilo Francisco da Silva Marçal

ORCID: https://orcid.org/0000-0002-9196-4424

Centro Universitário de Maringá, Brasil

E-mail: danilofsm@msn.com

Greice Westphal

ORCID: https://orcid.org/0000-0001-9107-0108

Universidade Estadual de Maringá, Brasil

E-mail: greicewes@gmail.com

Maria Luiza Costa Borim

ORCID: https://orcid.org/0000-0002-9523-4218

Universidade Estadual de Maringá, Brasil

E-mail: luborim10@hotmail.com

Cesar Faundez Casanova

ORCID: https://orcid.org/0000-0003-4501-4169 Universidad Católica del Maule, Chile E-mail: cfaundez@ucm.cl

Mario Moreira Castilho

ORCID: https://orcid.org/0000-0002-4855-8236

Universidade Estadual de Maringá, Brasil

E-mail: mmcastilho_1905@hotmail.com

Ricardo Souza de Carvalho

ORCID: https://orcid.org/0000-0003-1715-9213 Universidad Católica del Maule, Chile

E-mail: rsouza@ucm.cl

Nelson Nardo Junior

ORCID: https://orcid.org/0000-0002-6862-7868

Universidade Estadual de Maringá, Brasil

E-mail: nnjunior@uem.br

Sônia Cristina Dias Soares Vermelho

ORCID: https://orcid.org/0000-0003-2205-8070

Universidade Federal do Rio de Janeiro, Brasil

E-mail: cristina.vermelho@gmail.com

Flávio Bortolozzi

ORCID: https://orcid.org/0000-0003-0517-1127

Centro Universitário de Maringá, Brasil

E-mail: flavio.bortolozzi.53@gmail.com

\begin{abstract}
Resumo
Introdução: As mídias sociais, a academia e a pratica de musculação são fatores contribuintes para a influência da busca de um corpo perfeito na população jovem. Com a popularização das redes sociais, as musas fitness, blogueiros e modelos são grandes incentivadores para o consumo e venda de suplementos, vitaminas e anabolizantes, sem qualquer comprovação científica ou recomendação de um profissional de saúde Objetivo: nesta pesquisa, realizada com frequentadores de academia, buscamos analisar as relações entre motivação e expectativa do uso de esteroides anabolizantes e o envelhecimento. Métodos: Pesquisa de natureza aplicada e exploratória, com abordagem qualitativa,
\end{abstract}


utilizando como procedimento para a coleta de dados uma entrevista semiestruturada. Foram avaliados indivíduos usuários de esteroides anabolizantes do sexo masculino. Os dados foram extraídos através da análise de conteúdo. Resultados: participaram da pesquisa 18 indivíduos do sexo masculino, com média de idade de 28 anos; $72 \%$ são solteiros; a maioria com graduação e renda familiar variando de 4 a 10 mil reais. Quanto ao uso dos esteroides, os dados mostraram que sua utilização passa, inicialmente, de estéticos para um uso de natureza psicológica, pois os auxilia a superarem traumas passados. Consideramos que isso possa ser o motivo pelo qual os indivíduos não relacionaram o uso a consequências futuras (envelhecimento) mas como satisfação pessoal parcialmente momentânea. Conclusão: Os indivíduos buscam a academia devido ao fato de problemas de aceitação do corpo na infância ou na adolescência, buscando resultados rápidos para serem melhores visto na sociedade.

Palavras-chave: Academia; Envelhecimento; Esteroides anabolizantes.

\begin{abstract}
Introduction: The media, the gym and the practice of bodybuilding are contributing factors to the influence of the search for a perfect body in the young population. With the popularization of social networks, fitness muses, bloggers and models are great incentives for the consumption and sale of supplements, vitamins and anabolics, without any scientific evidence or recommendation from a health professional. Objective: in this research, carried out with gym goers, we sought to analyze the relationship between motivation and expectation of the use of anabolic steroids and aging. Methods: Research of an applied and exploratory nature, with a qualitative approach, using a semi-structured interview as a procedure for data collection. Individuals using male anabolic steroids were evaluated. The data were extracted through content analysis. Results: 18 male individuals participated in the research, with an average age of 28 years; $72 \%$ are single; the majority with graduation and family income varying from 4 to 10 thousand reais. As for the use of steroids, the data showed that their use goes, initially, from aesthetics to a use of a psychological nature, as it helps them to overcome past traumas. We consider this to be the reason why individuals did not relate the use to future consequences (aging) but as partly personal satisfaction. Conclusion: Individuals seek the gym due to the fact of problems with acceptance of the body in childhood or adolescence, seeking quick results to be better seen in society.
\end{abstract}

Keywords: Academia; Aging; Anabolic steroids.

\title{
Resumen
}

Introducción: Los medios de comunicación, el gimnasio y la práctica del culturismo son factores que contribuyen a la influencia de la búsqueda de un cuerpo perfecto en la población joven. Con la popularización de las redes sociales, las musas fitness, blogueras y modelos son grandes alicientes para el consumo y venta de suplementos, vitaminas y anabólicos, sin ninguna evidencia científica ni recomendación de un profesional de la salud. Objetivo: en esta investigación, realizada con asistentes al gimnasio, se buscó analizar la relación entre la motivación y la expectativa del uso de esteroides anabólicos y el envejecimiento. Métodos: Investigación de naturaleza aplicada y exploratoria, con enfoque cualitativo, utilizando una entrevista semiestructurada como procedimiento de recolección de datos. Se evaluaron los individuos que usaban esteroides anabólicos masculinos. Los datos se extrajeron mediante análisis de contenido. Resultados: participaron de la investigación 18 individuos del sexo masculino, con una edad promedio de 28 años; 72\% son solteros; la mayoría con graduación e ingresos familiares que varían de 4 a 10 mil reales. En cuanto al uso de esteroides, los datos muestran que su uso va, inicialmente, de la estética a un uso de carácter psicológico, ya que les ayuda a superar traumas pasados. Consideramos que esta es la razón por la que los individuos no relacionaron el uso con consecuencias futuras (envejecimiento) sino como satisfacción en parte personal. Conclusión: Los individuos acuden al gimnasio por problemas de aceptación del cuerpo en la niñez o adolescencia, buscando resultados rápidos para ser mejor vistos en la sociedad.

Palabras clave: Academia; Envejecimiento; Esteroides anabólicos.

\section{Introdução}

Essa busca pelo corpo "perfeito" tem sido motivo de preocupação entre as autoridades e os profissionais da saúde. Alguns estudos afirmam que o fato de vivermos em tempos atléticos em uma era estética faz com que o uso abusivo dos anabolizantes esteja relacionado com a insatisfação da aparência, baixa estima, pressão social, culto pelo corpo valorizado pela sociedade (Araújo, 2003; Cabral et al., 2020; Iriart et al., 2009)

As mídias, redes sociais a pratica de musculação são fatores contribuintes para a influência da busca de um corpo perfeito na população jovem. Com a popularização das redes sociais, as musas fitness, blogueiros e modelos são grandes incentivadores para o consumo e venda de suplementos, vitaminas e anabolizantes, sem qualquer comprovação científica ou recomendação de um profissional de saúde (Gomes et al., 2020; Rodrigues \& Chaves, 2016). 
A musculação é uma prática para obter qualidade de vida e bem-estar, os estudos apontam para os benefícios que essa atividade pode nos trazer quando inserida no nosso cotidiano, o que traz uma certa adversidade quando consideramos o uso de esteroides anabolizantes, que por sua vez são substâncias que derivam da testosterona que é um hormônio responsável pelos caracteres sexuais masculino biossíntese das proteínas e age catabolizando gorduras. Sua propriedade é indissociável o que traz características anabólicas e androgênicas (Sobrinho et al., 2020).

Os esteroides anabolizantes com finalidade de hipertrofia muscular e melhor desempenho durante a prática de exercício físico tem sido utilizados por frequentadores de academias, porém o abuso destas drogas pode causar diversas complicações no organismo, colocando em risco a própria vida, uma vez que nem sempre a indicação é feita por profissionais habilitados (Cabral et al., 2020; Faria et al., 2015).

A literatura tem demonstrado, que os usuários alegam utilizar essas drogas, pela preocupação com a estética e a maneira imediatista, com os recursos denominados esteroides anabolizantes e pela opção de custo baixo e acessível (Iriart et al., 2009; Sobrinho et al., 2020).

Diante disso, para a promoção da saúde é a preocupação pela maneira como são indicadas e adquiridas essas drogas. Existem relatos de que muitas vezes são "receitadas" por profissionais de Educação Física e/ou instrutores, e o acesso se dá de forma ilegal em farmácias, internet, exportações (Abrahin et al., 2013; Cabral et al., 2020; Iriart et al., 2009).

Em meio a este cenário, o objetivo geral dessa pesquisa foi analisar quais são as relações entre motivação e expectativa do uso de esteroides anabolizantes e o envelhecimento por frequentadores de academia. Além disso, objetivou-se, identificar o perfil dos usuários de esteroides anabolizantes, as expectativas e benefícios em relação aos exercícios físicos e ao uso de esteroides anabolizantes, além de investigar a relação que os usuários fazem entre o uso do produto e o envelhecimento.

\section{Metodologia}

Pesquisa de natureza aplicada, cujo objetivo foi de natureza exploratória, com abordagem qualitativa, utilizando como procedimento de pesquisa um roteiro semiestruturado. Foram avaliados indivíduos usuários de esteroides anabolizantes, do sexo masculino, onde os mesmos por meio de uma entrevista, puderam apontar suas opiniões com relação ao uso dos esteroides com finalidades estéticas.

Para o levantamento dos indivíduos alvos do estudo, foi realizada uma pesquisa informal nas academias da cidade de Maringá-Pr. O tamanho da amostra foi calculado a partir da aceitação dos entrevistados, o que se mostrou um fator que impôs grande dificuldade à realização da pesquisa pelo fato do uso de esteroides anabolizantes ser proibido e o uso para fins estéticos ser ilegal, muitos não aceitaram devido a desconfiança em relação a isso.

A entrevista foi realizada por telefone, respeitando a confidencialidade de cada entrevistado; sendo a mesma transcrita, identificando os núcleos temáticos e conceituais, uma vez que está transcrição facilitou a interpretação dos dados. O conteúdo foi tratado, a fim de identificar as recorrências, repetições e aspectos que mostraram comportamentos similares ou muito extravagantes em relação ao tema abordado.

Além das correlações, foi possível realizar a análise do conteúdo das entrevistas, compondo um contexto para os resultados das correlações. Após essa primeira etapa exploratória dos dados, foram realizadas as etapas de codificação textual, codificação conceitual, tratamentos e interpretação dos resultados.

A pesquisa foi autorizada pelo Comitê de Ética em Pesquisa (CEP), com aprovação de número1.571.382. Considerou-se como critérios de inclusão indivíduos do sexo masculino, usuários de esteroides anabolizantes, frequentadores de academias de musculação. A coleta de dados foi realizada no período de 20 de janeiro a 01 de março de 2017, de acordo com a disponibilidade dos participantes. 
A estrutura do instrumento de coleta de dados foi estruturada para obter os seguintes dados: idade, estado civil, profissão, moradia, Grau de Instrução, Renda Familiar. Em relação ao objeto de investigação, procuramos identificar: o papel da academia na vida dos indivíduos, a relação do uso de esteroides com o envelhecimento, e as perspectivas em relação ao uso de esteroides anabolizantes.

Os dados foram transcritos no editor de texto, organizados inicialmente em planilha de dados e apresentados por meio de tabelas, discursos coletivos e percentuais.

\section{Resultados}

Participaram desta pesquisa 18 indivíduos do sexo masculino, com média de idade de 28 anos, moradores da cidade de Maringá-PR, 72, \% são solteiros e possui graduação no ensino superior completo ou cursando, sendo que suas profissões são diversas e a renda familiar varia de 4 a 10 mil reais.

A análise do papel da academia na vida dos participantes desse estudo, demonstrou que a academia é um lugar onde pode-se encontrar amizade e ser aceito independente da pessoa, e em relação aos motivos que o fizeram praticar musculação foram estéticas, baixa autoestima (67\%), outros por praticar algum exercício físico (28\%), alguns por questões de saúde (22\%) e ainda se encontra os que utilizam a academia como forma de empreendimento (6\%).

Quando questionados sobre a função que o exercício físico tem em suas vidas atualmente, consideram- se que o mesmo é praticado por lazer (39\%), por ser uma parte fundamental de sua vida (22\%), por profissão $(22 \%)$, e até mesmo por saúde, esporte estética (11\%).

A questão sobre problemas passados com a aparência seja na infância e/ou na adolescência verificou-se que $67 \%$ dos entrevistados viveram de alguma maneira, o que pode ser constatado quando se pergunta qual seria hoje o ideal de porte físico e se depara com respostas como, aparência forte "ogro, monstro" (33\%), outros aponta que o ideal seria uma estética corporal (28\%), assim como corpo definido (22\%) ou apenas gostar do que vê (6\%).

Desses indivíduos participantes $94 \%$ acreditam que irão permanecer na musculação por muito tempo, devido a academia ter feito parte e ter mudado suas vidas em todos os sentidos.

Embora o envelhecimento ser um fator irreversível na vida do ser humano, esse fenômeno é pouco significativo para $61 \%$ dos entrevistados, pois eles não percebem o processo de envelhecimento e continuam com disposição, força e vitalidade, e os mesmos acreditam que envelhecer faz parte natural do processo de vida.

Dos 18 participantes da pesquisa 39\% não pensou sobre projetos para o envelhecimento, 28\% pretendem ter uma estabilidade financeira, $22 \%$ apontaram que o foco para o envelhecimento é ter cuidado com a saúde e outros $11 \%$ é manter um bom aspecto físico.

Partindo dessa resposta as medidas tomadas em relação ao envelhecimento ativo e saudável, obtivemos respostas que apontam que para os indivíduos usuários de esteroides anabolizantes o ideal para que se envelheça com saúde é praticar exercícios e cuidar da alimentação (50\%), não fumar, não beber, não se drogar (17\%) e alguns ainda acreditam que não é necessário manter nenhuma medida (33\%).

Portanto quando questionados ao que eles gostariam que a ciência inventasse sobre o envelhecimento $56 \%$ acreditam que envelhecer faz parte da vida, ou seja, é um processo natural, os outros apontam sugestões relacionadas a doenças, estéticas, alimentação e prolongamento da vida.

Para fazer a análise com relação ao uso de esteroides anabolizantes, questionou-se sobre onde os entrevistados obtiveram informações sobre o medicamento, e há quanto tempo estes fazem o uso, foi constatado que a média de utilização do grupo é de aproximadamente 6 anos, isto é, obteve-se nessa pesquisa indivíduos que fazem uso de 1 ano até os que usam há mais de 10 anos. 
Estes por sua vez apontaram que a academia foi a principal fonte de informação com relação aos esteroides, seguido pela internet e médicos sucessivamente, porém estes indivíduos adquirem o produto através de conhecidos $67 \%$, laboratórios e/ou farmácias, com receitas ilegais $45 \%$, através da internet, no mercado negro $17 \%$ e dentro das próprias academias $11 \%$, ou seja, embora $18 \%$ preferem os anabolizantes nacionais, devido a vigilância exigida para a comercialização, $12 \%$ usam de laboratórios específicos de sua confiança, $11 \%$ preferem os produtos importados por acreditar que estes sejam mais puros, e a maioria não tem preferência pela procedência do produto (59\%).

Investigou-se também quais foram os benefícios esperados pelos usuários que os levaram a fazer uso desses medicamentos, a maioria respondeu que eles procuravam resultados rápidos, no qual adquiriram em diversos aspectos como na melhora da performance e da auto estima, no aumento de massa e na libido, no rendimento, na estética.

Porém alguns entrevistados tem o conhecimento que o uso de esteroides para fins estéticos causam prejuízo a saúde, outros acreditam que depende do uso que é feito, tudo o que é abusivo se torna prejudicial, e ainda outros acreditam que não tem efeitos nenhum na saúde, mesmo diversos dos entrevistados ter tido efeitos colaterais devido ao uso dos esteroides como efeitos psíquicos (stress, irritação, insônia, depressão, síndrome do pânico, diminuição da imunidade, aumento da libido) e (físicos) calvície, engrossamento da voz, acne, dores nas pernas. Estes no entanto demostraram se preocupar com a saúde, porém esta preocupação não está relacionada ao envelhecimento.

Outro fator importante nesta pesquisa foi a questão da avaliação feita por amigos e familiares sobre o uso de esteroides, $55 \%$ respondeu que depende da pessoa, outros $28 \%$ apontaram que principalmente os familiares não sabem do uso, $11 \%$ são contra e apenas $6 \%$ entende e aceita esse fato.

Os entrevistados acreditam que o uso de esteroides vai contribuir para sua vida profissional, e social, devido eles acreditarem que a aparência é um fator importante para a sociedade atual, já as contribuições para vida afetiva 56\% acreditam que não irá fazer diferença, embora aumente a libido, o usuário pode ficar estressado, irritado, depressivo, sem paciência o que acaba por prejudicar a relação afetiva destes.

Mesmo os entrevistados se deparando com diversas controversas com relação ao uso de esteroides anabolizantes 39\% acredita que ao longo do tempo poderão ter problemas de saúde, 28\% não souber responder o que acreditam que possa resultar o uso prolongado, outros $17 \%$ apontaram que permaneceram ativos e viris, e ainda $17 \%$ acreditam que não terão problemas futuros, portanto encontrou-se que $67 \%$ dos entrevistados ainda almejam mudanças corporais, e $33 \%$ pretendem apenas manter o que conquistaram até hoje.

\section{Discussão}

Inicialmente pode-se encontrar com relação ao papel que a academia tem na vida dos usuários, fatores relacionados a estética, mudanças corporais, em geral motivado pelas relações interpessoais; bem como para treinamento para competições, e finalmente para melhorar sua qualidade de vida, também foram encontrados em estudos anteriores (Zamai et al., 2015).

Com o tempo, o ambiente da academia traz novos relacionamentos e o sentimento de amizade, sociabilização, onde fazer amigos ou encontrar amigos faz parte da rotina, são fatores motivacionais que estimulam a permanência ou a desistência dos praticantes. No grupo entrevistado alguns veem atividade física parte da vida, a maioria pretende pratica-la por muito tempo, entende-se que isso deve ao fato de terem observado os benefícios encontrados pelos treinamentos realizados (Tavares Junior \& Planche, 2017).

O espaço da academia acaba assumindo um papel fundamental em suas vidas, seja pelo cuidado com o corpo, ou como um lugar para se sociabilizar. É importante ressaltar que o motivo para a adesão à atividade física não está relacionado diretamente à saúde, mas pela busca do corpo perfeito, estimulado pela mídia e pela sociedade. Para nossos entrevistados os benefícios que a atividade proporciona torna-se fundamental em sua vida cotidiana, inicialmente pela estética, mas 
posteriormente também pela saúde, pela socialização, pelo bem estar ou prazer proporcionado pela prática de exercícios físicos. Esses aspectos foram encontrados em outros estudos (Cabral et al., 2020; Zamai et al., 2015), pois além de ser benéfica à saúde física, também está relacionada há preocupações com a aparência corporal, uma vez que se tornou uma preocupação na sociedade nos dias atuais (Cardoso \& Cardozo, 2014; Gomes et al., 2020).

Importante ressaltar, que a dimensão subjetiva relacionada ao stress, cansaço, também aparece relacionado à busca por atividade física, pois essa tem a função de proporcionar relaxamento mental. Já existem estudos que apontam que a atividade física traz diversos benefícios psicológicos, tais como melhorar o humor(Steffens et al., 2011; Westphal et al., 2020), diminuir a ansiedade, a depressão, em geral associado ao fato de que a melhora na imagem corporal aumenta a autoestima, proporcionando sensação de bem-estar; em consequência há diminuição do estresse e da tensão do dia a dia (Castilho et al., 2021; de Liz \& Andrade, 2016) . Portanto, inicialmente consideramos destacar a associação entre atividade física e benefícios físicos e psicológicos, assim diminuindo a negatividade frente a visão distorcida do corpo, tornando o corpo um ponto positivo, no qual acarreta em benefícios nos âmbitos social, físico e mental(Silva \& Ferreira, 2013).

O corpo é fator importante para estabelecermos relações com o mundo, através dele temos as primeiras sensações, ou seja, através do corpo é construído a relação com o mundo, e a imagem corporal está relacionada a estruturação da identidade do indivíduo na sociedade (Cardoso \& Cardozo, 2014). Demonstrando visibilidade em nosso estudo, pelo fato de vários entrevistados declararem que a não aceitação do corpo na infância ou na adolescência, foi um ponto crucial para a tomada de decisão de frequentar academia.

Peso acima de 100kg, muita massa muscular, pouca gordura, partes do corpo aumentado pela atividade física são ideais a serem conquistados nas academias. Existem fatores que influenciam direta e/ou indiretamente esta obsessão, onde destacamos o papel da mídia que estipula padrões de beleza, pois expõem diariamente imagens de homens com corpos excessivamente musculosos (Faria et al., 2015; Gomes et al., 2020). Em decorrência, a sociedade acaba incorporando esses modelos e passa a fazer "cobranças" por estes padrões; o que acaba induzindo a busca pela atividade física nas academias como o meio para intervir e realizar mudanças no corpo (Cardoso \& Cardozo, 2014; Silva \& Nunes, 2014).

Do ponto de vista da saúde, esses aspectos nos remetem a pensar sobre o processo de saúde ao longo da vida. Nesse sentido, em relação ao envelhecimento, 50\% dos entrevistados afirmam que não percebem o processo de envelhecimento, pois acreditam que a prática da atividade física e o modo de vida, retardam este processo. Interessante que alguns autores, realmente defendem que a atividade física serve como um fator para a prevenção de doenças crônico-degenerativas relacionadas ao envelhecimento (Sanchez et al., 2014). Evidenciam que o exercício físico atua sobre a desaceleração das alterações fisiológicas, e a idade biológica pode ser reduzida de 10 a 20 anos nos indivíduos praticantes de alguma atividade física em relação ao indivíduo não praticante (S. C. Santos \& Knijnik, 2009). Certamente, esse é um fator que também identificamos nas falas de nossos entrevistados e foi visto como um benefício decorrente da atividade física, porém certamente são associações que merecem ser aprofundadas, em especial porque nossos entrevistados são usuários de esteroides anabolizantes.

O envelhecimento são modificações morfológicas, fisiológicas, bioquímicas e psicológicas irreversíveis na vida do ser humano, as quais podem acarretar em diversas perdas progressivas nas diferentes capacidades do indivíduo (Cordeiro et al., 2014).Porém, interessante do grupo entrevistado foi que em geral não mostraram uma relação negativa com o envelhecimento, chegando mesmo a afirmar que envelhecer não é uma palavra bonita, que é "amadurecer". Na maioria das falas, o envelhecimento é um processo natural e a relação perante o envelhecimento foi de normalidade, ou com um sentimento positivo em relação a esse processo, contudo é importante ressaltar que essa questão também não foi motivo de preocupação constante. Talvez pela média de idade ou por outros fatores, essa relação frente ao envelhecimento contrasta com outras pesquisas em que as alterações devido a idade, em especial pelas limitações físicas, trazem um sentimento tristeza, de perda (Sanchez et al., 2014). 
As preocupações ao envelhecimento encontradas na pesquisa, estão relacionados aos cuidados com a saúde, vida estável financeiramente e com a estética, e o caminho para um envelhecimento saudável, está relacionado a boa alimentação e a prática da atividade física. Realmente, isso pode ser corroborado por estudos que apontam ser fundamental para se um envelhecimento com qualidade que não só o exercício físico, mas também a alimentação e hábitos saudáveis como ingestão alimentar correta, não fumar, atitudes que podem prevenir diversas doenças crônico-degenerativas vindos de hábitos de vida inadequados (Cardoso \& Cardozo, 2014; Sanchez et al., 2014).

Dos entrevistados $56 \%$ acreditam que o envelhecimento faz parte da vida e não gostaria que houvesse nenhum tipo de intervenção com relação ao envelhecimento, exceto com a cura de doenças e que os alimentos consumidos fossem melhorados. O que mostram dois campos de interesse para novas pesquisas sobre a relação saúde e envelhecimento.

Com relação aos esteroides, um ponto muito importante nesta pesquisa foi a evidencia encontrada em relação à forma de acesso as informações sobre o produto. Entre os usuários a internet, as academias e os próprios médicos, aparecem como as fontes de informação predominante sobre o produto, com forte evidência do papel da internet como a mais citada. Esse aspecto também foi encontrado em outra pesquisa que apontou que as trocas de informações sobre os anabolizantes ocorrerem através das redes sociais, abertamente em blogs da internet, em conversas entre os usuários e praticantes de musculação (Cabral et al., 2020; Gomes et al., 2020; Sanchez et al., 2014). O que por si só é um fator de grande preocupação, tanto pela falta de controle sobre a informação que existe na internet, quanto pela despreocupação dos usuários em relação ao consumo de um produto proibido por lei. Ou seja, isso nos indica que provavelmente não há controle nem fiscalização sobre esse produto, contudo pelas limitações do estudo, isso deverá ser melhor investigado.

Outro ponto interessante diz respeito à forma como adquirem; $94 \%$ afirmaram que adquirem o produto de "conhecidos", amigos ou de pessoas que traficam, seja por meio de contrabando, de compra em laboratórios, farmácias de manipulações, drogarias com receitas ilegais, em laboratórios caseiros e alguns até mesmo clandestinamente, pela internet. Esse aspecto nos indicou que, de alguma maneira parece haver a formação de "grupo" entre os consumidores, pois 100\% dos entrevistados afirmaram que muitos amigos, colegas, ou conhecidos fazem uso de esteroides anabolizantes, ou seja, alguns espaços de informação e de fornecimento acabam por criar e fortalecer uma rede de usuários, os quais mantem formas de acesso ilegal para que o grupo tenha a disposição o produto (Faria et al., 2015; Gomes et al., 2020).

As próprias academias comercializam o produto, mas também surgem os mercados negros, e até mesmo pessoas da área da saúde como médicos, farmacêutico ou enfermeiros que facilitam o acesso. Esse contexto de comercialização foi encontrado em outra pesquisa em que apareceram farmácias vendendo com receitas médicas falsificadas ou somente assinadas e entregues em branco possibilitando que o usuários faça sua prescrição no "mercado negro" sem orientações profissionais, mas somente de amigos ou mesmo nas lojas de suplementos (Balbino, 2015; De Quadros et al., 2010; Santos et al., 2012). Por se tratar de um comércio majoritariamente ilícito, a preocupação em relação a procedência do produto não emerge; ainda que $11 \%$ tenham expressado preferirem os produtos nacionais pelo fato de haver segurança no controle de qualidade e da vigilância sanitária, porém, em função da busca de resultados rápidos, $89 \%$ preferem os importados pois acreditam que são mais puros. Na literatura pesquisada, as preferências pela procedência dos esteroides indicaram as marcas Durateston $®$, Deca-durabolin $®$; Hemogenin ${ }^{\circledR}$, Deposteron, estes fabricados no Brasil e outros importados como Winstrol ${ }^{\circledR}$ da Espanha, Primobolan ${ }^{\circledR}$ do México/Espanha/Alemanha (Cecchetto et al., 2012).

Outro dado preocupante diz respeito ao tempo de uso. Nos pareceu um dado alarmante que a média encontrada no grupo foi de 6 anos de uso. Alguns estimulados pela busca de resultados mais rápidos, passaram a fazer uso dos esteroides próximo a 1 ano que estavam praticando musculação. Estimulados pelos resultados rápidos, para mantê-los faz-se necessário que o consumo se prolonga; confirmado por alguns entrevistados que afirmaram fazer uso há mais de 10 anos. Esse aspecto 
também foi evidenciado em outros estudos que o tempo de uso varia bastante, e que algumas pessoas o prolongam por muito tempo(Mateus, 2015), sem se preocupar com as consequências para a saúde a longo prazo.

Esse aspecto foi encontrado em nossa pesquisa, onde obtivemos respostas como: o uso de esteroides anabolizante pode manifestar diversos problemas de saúde, mas devido fazer parte de tratamento médico para deficiências androgênicas, o qual restabelece o funcionamento sexual como a libido e sensação de bem-estar, mantendo a virilizarão (Boff, 2010), os efeitos adversos são minimizados em prol da manutenção de uma condição sexualmente ativa e viril; e justificam citando que conhecem pessoas que usam há anos e hoje estão idosas e com a saúde estável.

É importante citar que essa relação entre uso dos esteroides e sexualidade (Dutra et al., 2012) é evidenciada, pois alguns de nossos entrevistados afirmaram que o uso ajudou nos relacionamentos amorosos devido ao aumento da libido, o aumento da autoestima, ainda que saibamos que a longo prazo pode trazer a diminuição na produção de esperma, disfunção erétil, redução de libido e podendo levar a infertilidade, e em casos mais graves a atrofia testicular (Cardoso \& Cardozo, 2014; Rodrigues \& Chaves, 2016). Certamente, as limitações desse estudo não permitem fazer grandes inferência, mas certamente é um bom tema para futuras investigações, aprofundar nessa relação entre uso de esteroides e sexualidade.

Sabemos que para se ter melhoras significativas de força e hipertrofia, deve-se fazer o uso de pelo menos $600 \mathrm{mg}$ de testosterona por semana (Mateus, 2015), porém outros estudos indicaram que a dosagem de 10 a 200 vezes mais alta que as dosagem terapêuticas podem afetar a função endócrina da testosterona (Dutra et al., 2012).

A expectativa para atingir o ideal estético, proporcionado pelo produto, ao trazer ganho de massa muscular em curto período de tempo devido ao acumulo de líquido na musculatura, aumenta a síntese proteica e consequentemente a síntese de massa, força e potência muscular (Balbino, 2015; Silva \& Ferreira, 2013). Dos entrevistados $89 \%$ são cientes de que o uso para fins estéticos pode fazer mal a saúde, em particular pelo uso indiscriminado dos esteroides que acarretam diversos males a saúde do indivíduo como inibição da produção de testosterona, alteração na pressão arterial, casos de hipertensão arterial, pode afetar a parte musculoesquelética, aumento de hematócrito, aumento prostático, aumento dos níveis de antígeno prostático específico (PSA) (Cecchetto et al., 2012).

Os problemas evidenciados pelos entrevistados foram irritação, stress, insônia, síndrome do pânico, depressão, dores de cabeça, diminuição da imunidade, aumento da libido, calvície, aumento de acne, dores nas pernas, engrossamento da voz, etc., o que já foi evidenciado por outras pesquisas como resultado do uso prolongado do produto (Maio, 2012; Basaria, 2010). Mas, além dos problemas de ordem física, os entrevistados, corroborando outra investigação, indicaram que esses sintomas ainda trazem prejuízos em relação a sociabilidade devido as alterações comportamentais e psicológicas, as quais vão desde esses sintomas identificados pelos entrevistados, até transtorno bipolar, psicose, o que pode levar ao isolamento e a depressão (Boff, 2010). Esses problemas de natureza relacional, também encontrado em nossa pesquisa quando a maioria dos entrevistados afirmam que o uso de esteroides não trouxe e nem irá trazer benefícios para a vida social (Goodman \& Gilman, 2012; Venâncio et al., 2010).

Nos ficou a impressão de que, ainda que tenham conhecimento dos efeitos colaterais e de sentirem esses efeitos, continuam tomando por razões muito fortes, para além dos motivos iniciais. Outros estudos também encontraram resultados em que os consumidores indicaram ter preocupações com a saúde, conhecerem os males que os anabolizantes podem acarretar na saúde, mas não se abstém do uso (Cecchetto et al., 2012). Ou seja, podemos indicar que não encontramos nesse grupo de entrevistados uma preocupação efetiva com a saúde e com o envelhecimento, pois mesmo com os benefícios inicialmente sentidos pela aceleração dos processos metabólicos, o que "retira" a sensação do envelhecimento; não avaliam os riscos em torno das probabilidades de tumores hepáticos, de aumento da pressão arterial, problemas vasculares, perdas ósseas, impotência dentre outros já mencionado anteriormente. Problemas que colocam em cheque os benefícios atuais, e que podem acarretar problemas futuros fazendo com que os indivíduos percam em qualidade de vida durante a velhice. Talvez por isso, a 
maioria afirmou que os familiares desconhecem o uso, pois sabem que podem ser recriminados, como realmente o são em alguns casos.

Pensando em promoção da saúde, em envelhecimento ativo, questionamos os indivíduos com relação as expectativas deles com relação ao uso de esteroides anabolizantes, o que o uso poderá contribuir para a vida profissional. Partindo da premissa de que a insatisfação com a imagem corporal é o principal motivo para o indivíduo fazer uso dessa substância, homens jovens já buscam um corpo ideal, pois o padrão de beleza, a imagem corporal, é tratada como sinônimo de saúde para se tornarem apresentáveis na sociedade. O que confirma que os padrões de belezas que vem sendo adquiridos nas últimas décadas, está fazendo com que a sociedade invista cada vez mais em modelo de beleza, reproduzindo a admiração pela visão de corpos fortes e musculosos, como estímulos para que busquem alcançar o corpo ideal (Cabral et al., 2020; Cecchetto et al., 2012; Gomes et al., 2020).

O custo individual e coletivo dessa busca, talvez ainda não possa ser dimensionado, pois somente daqui algumas décadas é que teremos uma geração de jovens usuários de esteroides anabolizantes e, nessa hora é que talvez possamos ter a real dimensão sobre a saúde pública das consequências sobre a saúde da população.

\section{Conclusão}

Como resultados dessa pesquisa, pode-se indicar que os indivíduos buscam a academia devido ao fato de problemas de aceitação do corpo na infância ou na adolescência, buscando resultados rápidos para serem melhores visto na sociedade.

Devido aos sentimentos positivos que os praticantes têm em relação a academia, esta passa ter papel fundamental em suas vidas, seja para o cuidado com a estética, para melhorar a aceitação do corpo e/ou para fazer amigos.Porém, a academia após um tempo passa a cumprir outras funções na vida desses indivíduos como lazer, forma de relaxamento, para desestressar, "terapia"; passa a ser um ambiente de relacionamento, para se fazer um ciclo de amizades, ou seja, podemos perceber que existe uma migração da dimensão estética (físico) para a dimensão subjetiva (bem-estar). Pensamos que os resultados alcançados nas práticas das atividades dentro da academia também é um fator para que haja motivação para que os indivíduos frequentem academia ao longo da vida.

Em relação aos esteroides anabolizantes, identificou-se que a internet é a principal fonte de informação, porém não se descarta a academia como um grande aliado, mesmo que os indivíduos buscam acompanhamentos médicos para o uso, atitude no qual não descarta os malefícios que os esteroides podem causar, seja pelo uso prolongado, seja pela forma inadequada em termos de dosagem, seja pela procedência da substância.

Identificamos também nesta pesquisa que a busca por resultados rápidos, mais especificamente para aumentar a massa muscular, diminuir gordura, para aumentar a força física, para dar melhor condicionamento físico e definição corporal, faz com que os pesquisados utilizem substâncias ilícitas, mesmo cientes dos males que essas drogas causam à saúde, os resultados alcançados prevalecem na decisão de fazer o uso dos esteroides anabolizantes, mesmo que preocupados com a saúde, muitos não relacionam os cuidados atuais com os prejuízos e as consequências na terceira idade.

E por fim, quanto ao processo de envelhecimento, constatou-se que os entrevistados não percebem em si o processo de envelhecimento, talvez pela disposição, força e vitalidade, e que os mesmo acreditam que envelhecimento seja um processo normal, mesmo que alguns sentem as limitações, não projetam expectativas futuras com relação ao envelhecimento, demonstrado pelos poucos conhecimentos mostrados em relação aos cuidados necessários para um envelhecimento de qualidade, ou seja, percebemos um desconhecimento dos indivíduos com relação ao envelhecimento saudável.

Em relação as dimensões da vida, acredita-se que a estética corporal pode trazer benefícios para a vida profissional, no qual alguns ainda almejam mudanças corporais. Porém, na vida social, os mesmos não acreditam que possam trazer grandes 
contribuições. Porém é importante evidenciar que em relação a afetividade há divergências, pois alguns acreditam que pode ajudar, mas isso não é consenso.

Mesmo conhecendo os prejuízos que o uso de anabolizante pode acarretar à saúde a longo prazo, os mesmos não têm intenções em interromper o uso, pois a utilização serve para superar problemas relacionados a aceitação corporal.

Finalizando, para se promover a saúde é fundamental que as pessoas tenham consciência dos cuidados básicos hoje para que se tenha um envelhecimento saudável, a busca por resultados rápidos, podem comprometer o futuro de maneira permanente.

\section{Agradecimentos}

Agradeço este artigo ao professor Doutor Diógenes Aparício Garcia Cortez que infelizmente no decorrer da pesquisa faleceu por questões de saúde ao professor Doutor Nelson Nardo Junior que acredita em meu trabalho acadêmico e indiretamente me auxiliou na produção do artigo e a minha família por todo apoio e credibilidade depositada em mim.

\section{Referências}

Abrahin, O. S. C., Souza, N. S. F., Sousa, E. C. de, Moreira, J. K. R., \& Nascimento, V. C. do. (2013). Prevalência do uso e conhecimento de esteroides anabolizantes androgênicos por estudantes e professores de educação física que atuam em academias de ginástica. Revista Brasileira de Medicina do Esporte, 19(1), 27-30. https://doi.org/10.1590/s1517-86922013000100005

Araújo, J. (2003). O Uso De Esteroides Androgênicos Anabolizantes Entre Estudantes Do Ensino Médio No Distrito Federal. 1-90.

Balbino, G. (2015). Insatisfação corporal e o uso de esteróides anabólicos em homens praticantes de musculação. Aleph.

Basaria. (2010). Androgen abuse in athletes: Detection and consequences. Journal of Clinical Endocrinology and Metabolism, 95(4),1533-1543. http://www.embase.com/search/results?subaction=viewrecord\&from=export\&id=L359230762\%5Cnhttp://jcem.endojournals.org/cgi/reprint/95/4/1533\%5Cnh ttp://dx.doi.org/10.1210/jc.2009-1579\%5Cnhttp://sfx.library.uu.nl/utrecht?sid=EMBASE\&issn=0021972X\&id=doi:10.1210\%2Fjc.200

Boff, S. R. (2010). Esteróides Anabólicos e Exercício: Ação e Efeitos Colaterais. Revista Brasileira de Ciência e Movimento, 18(1), 81-88. https://doi.org/10.18511/0103-1716/rbcm.v18n1p81-88

Cabral, P. R. F., Nascimento, J. G. N. Do, Nunes, J. R. D. S., \& Pereira Júnior, J. C. (2020). O uso de anabolizantes e suplementos alimentares por frequentadores de uma academia no município de Cuiabá - MT sob o olhar da biomedicina. Research, Society and Development, 9(11), e1159119531. https://doi.org/10.33448/rsd-v9i11.9531

Cardoso, D. S., \& Cardozo, M. A. V. (2014). Corpo, contemporaneidade e representações: academia porque? Omnia Saúde, 11(1), 58-80. www.fai.com.br

Castilho, M. M., Westphal, G., Thon, R. A., Pereira, I. A. S., Martins, F. M., Amaral, M. F. do, Okawa, R. T. P., \& Nardo Junior, N. (2021). Efeitos de um programa multiprofissional de tratamento da obesidade no ambiente aquático em adultos com obesidade severa. Research, Society and Development, 10(1), e12910111636. https://doi.org/10.33448/rsd-v10i1.11636

Cecchetto, F., de Moraes, D. R., \& de Farias, P. S. (2012). Distintos enfoques sobre esteroides anabolizantes: Riscos à saúde e hipermasculinidade. Interface: Communication, Health, Education, 16(41), 369-382. https://doi.org/10.1590/S1414-32832012005000008

Cordeiro, J., Del Castillo, B. L., Freitas, C. S. de, \& Gonçalves, M. P. (2014). Efeitos da atividade física na memória declarativa, capacidade funcional e qualidade de vida em idosos. Revista Brasileira de Geriatria e Gerontologia, 17(3), 541-552. https://doi.org/10.1590/1809-9823.2014.13006

de Liz, C. M., \& Andrade, A. (2016). Análise qualitativa dos motivos de adesão e desistência da musculação em academias. Revista Brasileira de Ciencias do Esporte, 38(3), 267-274. https://doi.org/10.1016/j.rbce.2015.11.005

De Quadros, T. M. B., Gordia, A. P., Martins, C. R., Silva, D. A. S., Ferrari, E. P., \& Petroski, É. L. (2010). Imagem corporal em universitários: Associação com estado nutricional e sexo. Motriz. Revista de Educacao Fisica, 16(1), 78-85. https://doi.org/10.5016/1980-6574.2010v16n1p78

Dutra, B. S. C., Pagani, M. M., \& Ragnini, M. P. (2012). Esteróides Anabolizantes: Uma Abordagem Teórica. Revista Científica da Faculdade de Educação e Meio Ambiente, 3(2), 21-39.

Faria, J. G. De, Cabral, S. A., Silva, T. V. da, \& Miyamoto, C. A. (2015). Esteroides anabolizantes : Culto ao corpo e seus principais efeitos sobre o organismo Revista Conexão Eletrônica, 12(1), 1-8.

Gomes, C. A. C., Fecury, A. A., Dendasck, C. V., Dias, C. A. G. de M., Moraes, J. S., Moreira, E. C. de M., Cruz Neto, M. S. da, Santos, D. L., Souza, K. O. da, Silva, I. R. da, Araújo, M. H. M. de, Pinheiro, M. da C. N., \& Oliveira, E. de. (2020). Suplementação nutricional e sua associação com a saúde e a composição corporal de praticantes de musculação. Research, Society and Development, 9(9), e877997962. https://doi.org/10.33448/rsd-v9i9.7962

Goodman, \& Gilman. (2012). As Bases Farmacológicas da Terapeutica de Goodman e Gilman.

Iriart, J. A. B., Chaves, J. C., \& Orleans, R. G. de. (2009). Culto ao corpo e uso de anabolizantes entre praticantes de musculação. Cadernos de Saúde Pública, 25(4), 773-782. https://doi.org/10.1590/s0102-311x2009000400008 
Research, Society and Development, v. 10, n. 6, e22010615735, 2021

(CC BY 4.0) | ISSN 2525-3409 | DOI: http://dx.doi.org/10.33448/rsd-v10i6.15735

Maio, P. (2012). Manifestações cutâneas do uso de esteroides anabolizantes em atletas de alta competição. 3(4), 13-14.

Mateus, H. C. (2015). Fatores motivacionais e o uso de esteroides anabolizantes por homens praticantes de musculação em academias da região do grande pinheirinho no município de Criciúma, SC. 16p.

Rodrigues, A. L. de P., \& Chaves, R. de F. (2016). Consumo de suplementos alimentares por praticantes de musculação em uma academia de Fortaleza-CE. RBNE - Revista Brasileira de Nutrição Esportiva, 10(60), 597-602.

Sanchez, M. A., Brasil, J. M. M., \& Ferreira, I. A. M. (2014). Benefícios de um programa de atividade física para a melhoria da qualidade de vida de idosos no estado do Rio de Janeiro. Revista Brasileira de Ciências do Envelhecimento Humano, 11(3). https://doi.org/10.5335/rbceh.2014.4528

Santos, N. D. O., Marques, V. G., Maihara, A., Rosana, G., Benute, G., Cristina, M., \& Lucia, S. De. (2012). Vigorexia , Uso De Anabolizantes E a (Não) Procura Por Tratamento Psicológico: Relato De Experiência. Psicologia Hospitalar, 10(1), 2-15.

Santos, S. C., \& Knijnik, J. D. (2009). Motivos de adesão à prática de atividade física na vida adulta intermediária. Revista Mackenzie de Educação Física e Esporte, 5(1).

Silva, D., \& Nunes, H. (2014). Imagem corporal e estágios de mudança de comportamento para atividade física em universitários. Revista Brasileira de Atividade Física \& Saúde, 19(5). https://doi.org/10.12820/rbafs.v.19n5p597

Silva, R. S., \& Ferreira, V. L. (2013). Avaliação Da Auto-Imagem Em Frequentadores De Academia No Interior Do Rio Grande Do Sul Resumo. Revista de Psicologia, 16(24), 23-30. https://revista.pgsskroton.com/index.php/renc/article/view/2452

Sobrinho, C. A., Rossi Junior, W. C., Corsini, W., Soares, E. A., \& Esteves, A. (2020). Doses suprafisiológicas de esteroides anabolizantes e os efeitos no coração de ratos jovens sedentários: estudo morfométrico. Research, Society and Development, 9(11), e72091110079. https://doi.org/10.33448/rsdv9i11.10079

Steffens, R. de A. K., Liz, C. M. de, Viana, M. da S., Brandt, R., Oliveira, L. G. A. de, \& Andrade, A. (2011). Praticar caminhada melhora a qualidade do sono e os estados de humor em mulheres com síndrome da fibromialgia. Revista Dor, 12(4), 327-331. https://doi.org/10.1590/s1806-00132011000400008

Tavares Junior, A. C., \& Planche, T. C. (2017). Motivos de Adesão de Mulheres a Prática de Exercícios Físicos em Academias. Revista Equilíbrio Corporal e Saúde, 8(1), 28. https://doi.org/10.17921/2176-9524.2016v8n1p28-32

Venâncio, D. P., Nóbrega, A. C. L. da, Tufik, S., \& Mello, M. T. de. (2010). Avaliação descritiva sobre o uso de esteroides anabolizantes e seu efeito sobre as variáveis bioquímicas e neuroendócrinas em indivíduos que praticam exercício resistido. Revista Brasileira de Medicina do Esporte, 16(3), 191-195. https://doi.org/10.1590/s1517-86922010000300007

Westphal, G., Baruki, S. B. S., Mori, T. A. de, Montebello, M. I. de L., \& Pazzianotto-Forti, E. M. (2020). Effects of Individualized Functional Training on the Physical Fitness of Women with Obesity. Lecturas: Educación Física y Deportes, 25(268), 61-75. https://doi.org/10.46642/efd.v25i268.2084

Zamai, C. A., Filocomo, M., \& Rodrigues, A. A. (2015). Qualidade de Vida, Diversidade, Sustentabilidade (P. Editorial (org.). 